\title{
Cognitive and Neural Aspects in Robotics with Applications
}

\author{
Madan M. Gupta, ${ }^{1}$ Noriyasu Homma, ${ }^{2}$ Zeng-Guang Hou, ${ }^{3}$ and Ivo Bukovsky ${ }^{4}$ \\ ${ }^{1}$ Intelligent Systems Research Laboratory, College of Engineering, University of Saskatchewan, SK, Canada S7N 5A9 \\ ${ }^{2}$ Tohoku University, Sendai, Japan \\ ${ }^{3}$ Chinese Academy of Sciences, Beijing, China \\ ${ }^{4}$ Czech Technical University, Prague, Czech Republic
}

Correspondence should be addressed to Madan M. Gupta, madan.gupta@usask.ca

Received 25 August 2010; Accepted 25 August 2010

Copyright (C) 2010 Madan M. Gupta et al. This is an open access article distributed under the Creative Commons Attribution License, which permits unrestricted use, distribution, and reproduction in any medium, provided the original work is properly cited.

With the evolution of our complex technological society and the introduction of new notions and innovative theoretical tools such as cognitive and neural aspects in the field of robotics systems, this has brought some new evolutions. These evolving and theoretical tools are providing some intelligence and robustness in robotics systems similar to those found in biological species.

Cognition and intelligence-the ability to learn, understand, and adapt - is the creation of nature, and it plays a key role in human actions and in many other biological species. Humans possess some robust attributes of learning and adaptation, and that is what makes them so intelligent. We humans react through the process of learning and adaptation on information received through a widely distributed network of sensors and control mechanisms in our body. The faculty of cognition, which is contained in our carbon-based computer, the brain, acquires information from the environment through various sensory mechanisms such as vision, hearing, touch, taste, and smell. Then, the process of cognition, through its intricate neural networksthe cognitive computing-integrates this information and provides appropriate actions. The cognitive process then advances further towards some attributes such as learning, recollection, reasoning, and control.

We are learning from the carbon-based cognitive computer - the brain - and now are in the process of inducing two important aspects of perception and cognition, and thereby that of the intelligence into robotics machines. One of our aims is to construct a robotic vehicle that can think and operate in uncertain and unstructured driving conditions. Robots in manufacturing, mining, agriculture, space and ocean exploration, and health sciences are just a few examples of these challenging applications where humanistic attributes such as cognition and intelligence can play an important role.

The proposal for the special issues of the robotics journal devoted to cognitive and neural aspects was conceived in early 2009, and now we are pleased to present 18 invited research papers which cover a wider aspect of cognition and intelligence. Initially, we received 29 research papers, but after going through a thorough review process, we have accepted only 18 papers. These papers cover some wider aspects of cognition and intelligence in the field of robotics, and for this special issue of the Robotics Journal, we have divided them into the following three parts.

In the first part, the first six papers (1-6) cover the fields of cognition, perception, vision, and neural learning in robotics. In the second part, the second six papers (7-12) deal with neuro-control in robotics. Finally, in the third part, the last six papers (7-18) focus on miscellaneous robotics applications.

Thus, as can be seen, these 18 invited papers represent a broad cross-section of the robotics field and have used some cognitive and neural aspects in the design of learning and control algorithms. Also, these papers have been authored by 55 researchers in robotics field from 29 different research institutions or universities located in seven different countries (Austria (1), Canada (1), China (4), India (1), Japan (8), Sweden (2), and USA (3)).

We guest editors believe that this special issue of the Journal of Robotics containing 18 invited research papers authored by international researchers and devoted to the 
various aspects of cognition, perception, vision and neural learning, and neuro-control in robotics with various industrial applications is an informative and useful addition to the field of robotics.

\section{Acknowledgments}

We the Guest Editors would like to express their sincere appreciation to the Editorial Board of the Journal of Robotics for their confidence and unwavering support for this special issue on "Cognitive and Neural Aspects in Robotics with Applications." We acknowledge the efforts of the authors for their valuable research contributions to this special issue of the journal, and that of the reviewers who adhered to the strict timeline for making this special issue a success.

Madan M. Gupta Noriyasu Homma Zeng-Guang Hou Ivo Bukovsky 

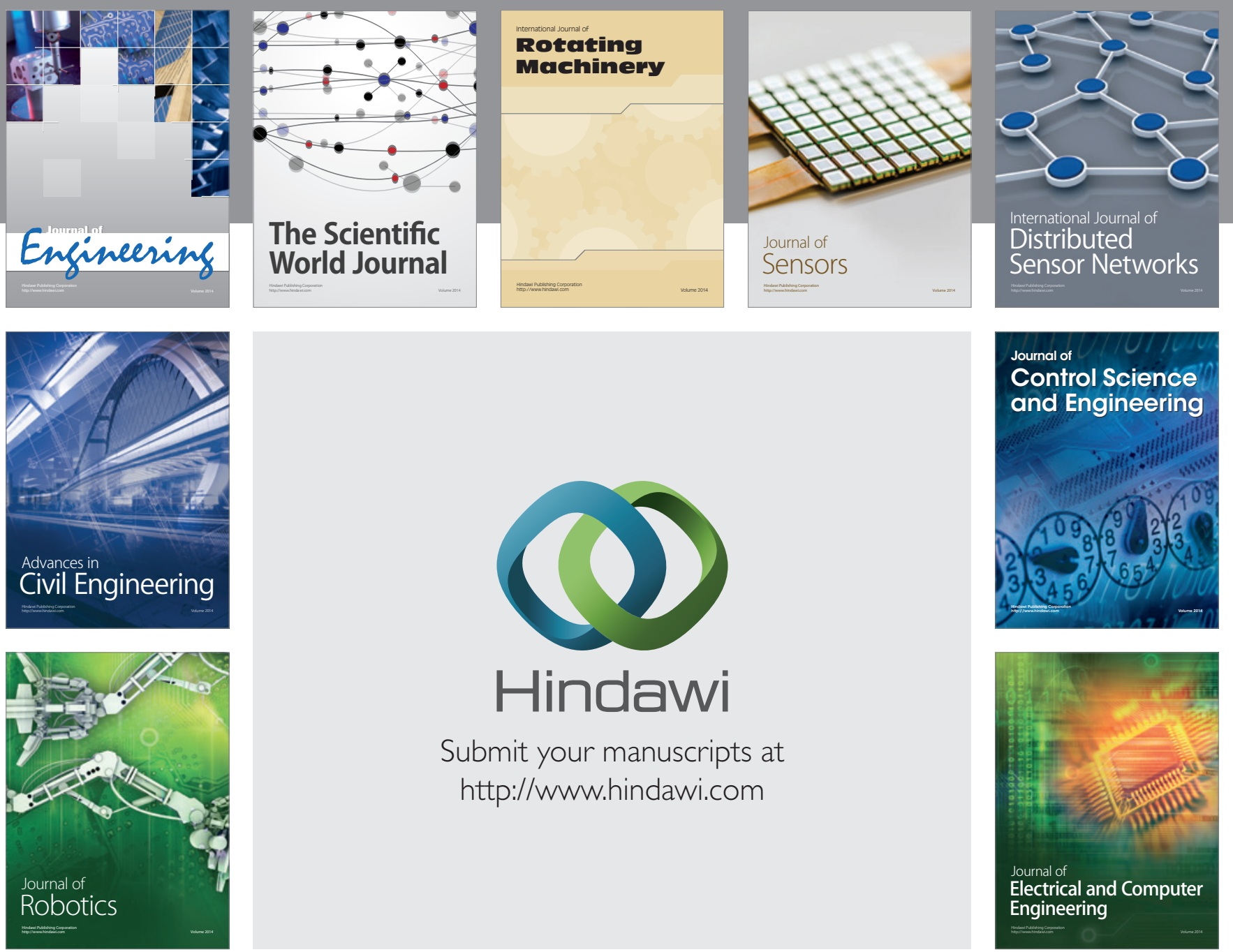

Submit your manuscripts at

http://www.hindawi.com
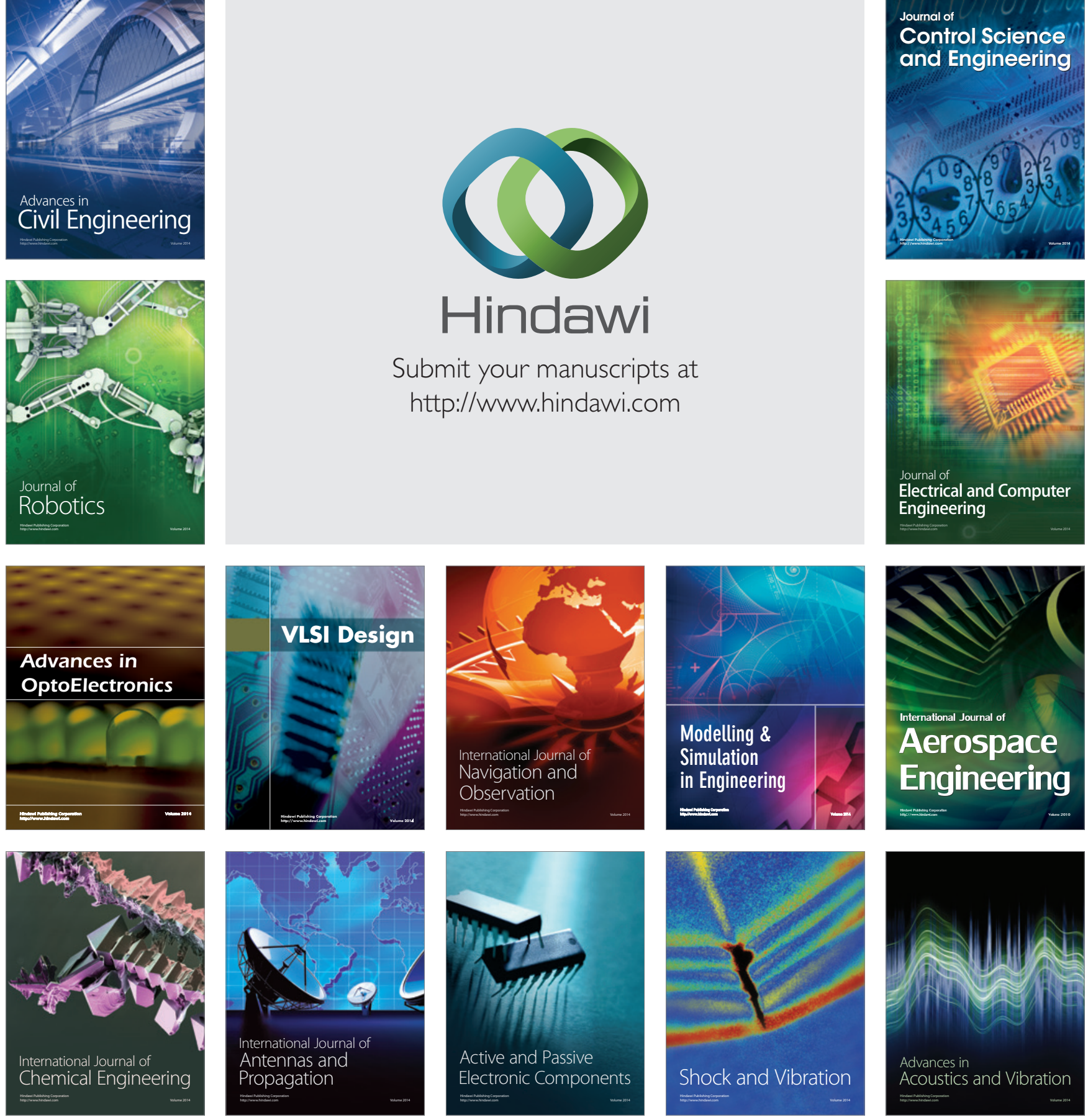